\title{
Noninvasive indices of arterial stiffness in hemodialysis patients
}

\author{
Kiyo Ueyama ${ }^{1}$, Masaaki Miyata ${ }^{1}$, Takuro Kubozono ${ }^{1}$, Aya Nagaki ${ }^{1}$, Shuichi Hamasaki ${ }^{1}$, Satonori Ueyama ${ }^{2}$
} and Chuwa Tei ${ }^{1}$

The purpose of this study was to evaluate the validity of brachial-ankle pulse wave velocity (baPWV) and the cardio-ankle vascular index (CAVI) as measures of arterial stiffness in hemodialysis (HD) patients. We studied 160 consecutively enrolled HD patients (mean age: $59 \pm 13$ years; 91 male patients). We measured baPWV and CAVI using a VaSera VS-1000, maximum intima-media thickness (max IMT) of the carotid artery by ultrasonography and blood renal and lipid parameters. As a control, baPWV and CAVI were also measured in age- and gender-matched healthy volunteers. Both baPWV and CAVI were significantly higher in HD patients than in controls (baPWV: $1698 \pm 355$ vs. $1454 \pm 263 \mathrm{~cm} \mathrm{~s}^{-1}, P<0.0001$; CAVI: $9.3 \pm 1.4$ vs. $8.9 \pm 1.2$, $P<0.01)$. BaPWV correlated positively with age $(r=0.549, P<0.0001)$, systolic blood pressure (SBP) $(r=0.510, P<0.0001)$, diastolic blood pressure $(r=0.203, P<0.0001)$, pulse pressure (PP) $(r=0.499, P<0.0001), \mathrm{Kt} \mathrm{V}^{-1}(r=0.221, P<0.01)$, Brinkman index $(r=0.186, P<0.05)$ and max IMT $(r=0.285, P<0.001)$. CAVI also correlated positively with age $(r=0.562, P<0.0001)$, SBP $(r=0.395, P<0.0001)$, PP $(r=0.490, P<0.0001), \mathrm{KtV}^{-1}(r=0.216, P<0.01)$, Brinkman index $(r=0.238, P<0.01)$ and max IMT $(r=0.280, P<0.001)$. Multiple regression analysis demonstrated baPWV and CAVI correlated independently with age and SBP. Receiver operating characteristics (ROC) curve analysis demonstrated that baPWV and CAVI had similar power to predict increases in max IMT. We also measured baPWV and CAVI immediately before and after HD, and showed CAVI was influenced by changes in water volume. Both baPWV and CAVI are therefore useful indices of arterial stiffness in HD patients.

Hypertension Research (2009) 32, 716-720; doi:10.1038/hr.2009.83; published online 12 June 2009

Keywords: arterial stiffness; baPWV; CAVI; hemodialysis

\section{INTRODUCTION}

Chronic kidney disease is a worldwide public health problem and is a risk factor for the development of cardiovascular disease. ${ }^{1}$ The incidence and associated costs of treatment of end-stage renal disease are increasing in Japan similar to that occurring in Western countries. ${ }^{2,3}$ Accelerated atherosclerosis in end-stage renal disease patients is a serious problem and the resulting poor prognosis in hemodialysis (HD) patients is an important issue. ${ }^{4,5}$

Brachial-ankle pulse wave velocity (baPWV) is used as a noninvasive clinical index of arterial stiffness ${ }^{6,7}$ and has been shown to predict the presence of coronary artery disease ${ }^{8}$ and also correlate with abdominal aortic calcification ${ }^{9}$ and carotid intima-media thickness. ${ }^{10}$ Although baPWV is a useful index for measuring arterial stiffness in HD patients, ${ }^{11-14}$ it has the limitation of being influenced by changes in blood pressure (BP) during measurements.

Recently, an atherosclerotic index, the cardio-ankle vascular index (CAVI), has been developed that involves measuring pulse wave velocity (PWV) and BP. CAVI is adjusted for BP based on the stiffness parameter $\beta$ and therefore measures arterial stiffness independent of
BP. ${ }^{15-17}$ We have reported previously that CAVI showed a weaker correlation with systolic BP (SBP) than baPWV and was not affected by changes in BP during measurement. ${ }^{9}$ There is also evidence that CAVI reflects histological arterial fibrosis and is a useful clinical marker for evaluating arterial stiffness in HD patients. ${ }^{18}$ The purpose of the study was therefore to evaluate the validity of the noninvasive indices of arterial stiffness, baPWV and CAVI, in HD patients.

\section{METHODS}

Study subjects

We analyzed 160 consecutively enrolled patients with kidney failure who received HD therapy at Ueyama Hospital. The causes of kidney failure were nephritis in 120 patients, diabetes in 32 patients, collagen disease in seven patients and Fabry disease in one patient. Of these patients, 42 were treated with an angiotensin II receptor blocker (ARB), four with an angiotensinconverting enzyme inhibitor and 14 with a HMG-CoA reductase inhibitor. Exclusion criteria were diseases that affect baPWV and CAVI measurements including the presence of atrial fibrillation, a high frequency of ventricular and atrial premature beats, peripheral arterial disease with an ankle-brachial

${ }^{1}$ Department of Cardiovascular, Respiratory and Metabolic Medicine, Graduate School of Medicine, Kagoshima University, Kagoshima, Japan and ${ }^{2} \mathrm{Jinaikai}$ Ueyama Hospital, Kagoshima, Japan

Correspondence: Dr M Miyata, Department of Cardiovascular, Respiratory and Metabolic Medicine, Graduate School of Medicine, Kagoshima University, 8-35-1 Sakuragaoka, Kagoshima 890-8520, Japan.

E-mail: miyatam@m3.kufm.kagoshima-u.ac.jp

Received 24 December 2008; revised 12 May 2009; accepted 13 May 2009; published online 12 June 2009 
pressure index (ABI) of less than 0.9, heart failure (New York Heart Association class II and higher), pulmonary edema and cancer. To compare baPWV and CAVI between the HD patients and control subjects, 160 healthy volunteers matched with the HD patients for age and gender were enrolled in the study.

The protocol of this study was approved by the Institutional Review Board of Kagoshima University. Informed consent was given by all the patients and volunteers.

\section{Measurements of baPWV and CAVI}

Immediately before HD, baPWV and CAVI were measured using a Vasera VS1000 (Fukuda Denshi, Tokyo, Japan). As reported previously, cuffs were placed on both ankles and the brachium, which were not used for blood access. Electrocardiographic electrodes were attached to the upper arm and a microphone was placed on the sternal angle for phonocardiography. The subjects rested in the supine position for $5 \mathrm{~min}$. PWV was calculated by dividing the distance from the aortic valve to the ankle artery by the sum of the difference between the time the pulse waves were transmitted to the brachium and the time the same waves were transmitted to the ankle, and the time difference between the second heart sound on the phonocardiogram and the notch of the brachial pulse waves. ${ }^{15-17}$ To minimize cuff inflation effects on blood flow dynamics, pulse waves were measured with the cuffs inflated to lower than diastolic pressure $(50 \mathrm{~mm} \mathrm{Hg})$. The extremity blood pressure was then measured by oscillometry. SBP, diastolic BP (DBP) and pulse pressure (PP) were obtained by measuring the $\mathrm{BP}$ at the right brachial artery.

CAVI was calculated by the following equation: CAVI $=a[\{2 \rho \times 1 /$ $(\mathrm{SBP}-\mathrm{DBP})\} \times\left\{\right.$ In $\left.\left.(\mathrm{SBP} / \mathrm{DBP}) \times \mathrm{PWV}^{2}\right\}\right]+b$ ( $\rho$ : density of blood, $a$ and $b$ : constants). ${ }^{16,17,19}$

\section{Analysis of blood samples}

Blood samples were obtained after an overnight fast on the morning of the day $\mathrm{baPWV}$ and CAVI were measured. The serum concentrations of creatinine $(\mathrm{Cr})$, blood urea nitrogen (BUN), uric acid (UA), calcium $(\mathrm{Ca})$, phosphoric acid $(\mathrm{P})$, parathyroid hormone (PTH), total cholesterol, triglyceride (TG) and highdensity lipoprotein cholesterol (HDL-C) were measured using standard laboratory procedures. Low-density lipoprotein cholesterol (LDL-C) was calculated by the Friedewald equation. $\mathrm{Kt} \mathrm{V}^{-1}$ was determined according to the procedure of Shinzato et al. ${ }^{20}$

\section{Measurement of maximum IMT}

The maximum IMT (max IMT) of the carotid artery was evaluated by high-resolution ultrasonography (Fukuda Denshi) using a 7.5-MHz probe. The IMT was measured as the distance from the leading edge of the first echogenic line to the leading edge of the second echogenic line. In this study, the thickest point of the bilateral carotid artery was defined as the max IMT without plaque.

\section{Statistical analysis}

Data are expressed as the mean \pm s.d. Differences between the mean values of the two groups were analyzed by unpaired $t$-tests, whereas differences between mean values of measurements at two points were analyzed using paired $t$-tests. The relationship between continuous variables was analyzed by linear regression analysis and independent associations between variables were examined by multiple regression analysis. The statistical analyses were performed with Statview version 5.0 (SAS Institute, Cary, NC, USA), whereas receiver operating characteristics (ROC) curve analysis was performed with JMP version 5.1.1 (SAS Institute). $P$-values less than 0.05 were considered statistically significant.

\section{RESULTS}

\section{Comparison of baPWV and CAVI between HD patients and} controls

The clinical characteristics of the HD patients and age- and gendermatched control subjects are summarized and compared in Table 1.

Table 1 Clinical characteristics of the control and HD groups

\begin{tabular}{lccc}
\hline Variable & Control $(\mathrm{n}=160)$ & $H D(\mathrm{n}=160)$ & P-value \\
\hline Age (years) & $60 \pm 10$ & $60 \pm 13$ & $\mathrm{NS}$ \\
Gender (M/F) & $91 / 69$ & $91 / 69$ & $\mathrm{NS}$ \\
BMI (kg m ${ }^{-2}$ ) & $23.2 \pm 2.8$ & $21.6 \pm 7.1$ & $<0.05$ \\
SBP (mm Hg) & $127 \pm 14$ & $148 \pm 22$ & $<0.0001$ \\
DBP (mm Hg) & $83 \pm 10$ & $87 \pm 13$ & $<0.001$ \\
PP & $45 \pm 9$ & $62 \pm 17$ & $<0.001$ \\
Cr (mg per 100 ml) & $0.8 \pm 0.2$ & $11.0 \pm 3.0$ & $<0.0001$ \\
BUN (mg per 100 ml) & $16 \pm 4$ & $71 \pm 16$ & $<0.0001$ \\
UA (mg per 100 ml) & $5.2 \pm 1.3$ & $6.8 \pm 1.4$ & $<0.0001$ \\
HDL-C (mg per 100 ml) & $57 \pm 14$ & $54 \pm 17$ & $\mathrm{NS}$ \\
LDL-C (mg per 100 ml) & $130 \pm 31$ & $80 \pm 27$ & $<0.0001$ \\
TG (mg per 100 ml) & $112 \pm 69$ & $104 \pm 62$ & $\mathrm{NS}$ \\
Duration of dialysis (days) & - & $3138 \pm 2498$ & $\mathrm{ND}$ \\
& & & \\
Medication & & & $\mathrm{ND}$ \\
$\quad$ ARB & 0 & 42 & $\mathrm{ND}$ \\
ACE-I & 0 & 14 & $\mathrm{ND}$ \\
Statin & 0 & 48 & \\
\hline
\end{tabular}

Abbreviations: ACE-I, angiotensin-converting enzyme inhibitor; ARB, angiotensin II receptor blocker; BMI, body mass index; BUN, blood urea nitrogen; $\mathrm{Cr}$, serum creatinine; DBP, diastolic blood pressure; HD, hemodialysis; HDL-C, high-density lipoprotein cholesterol; LDL-C, lowdensity lipoprotein cholesterol; ND, not done; NS, not significant; PP, pulse pressure; SBP, systolic blood pressure; statin, HMG-CoA reductase inhibitor; TG, triglyceride; UA, uric acid.
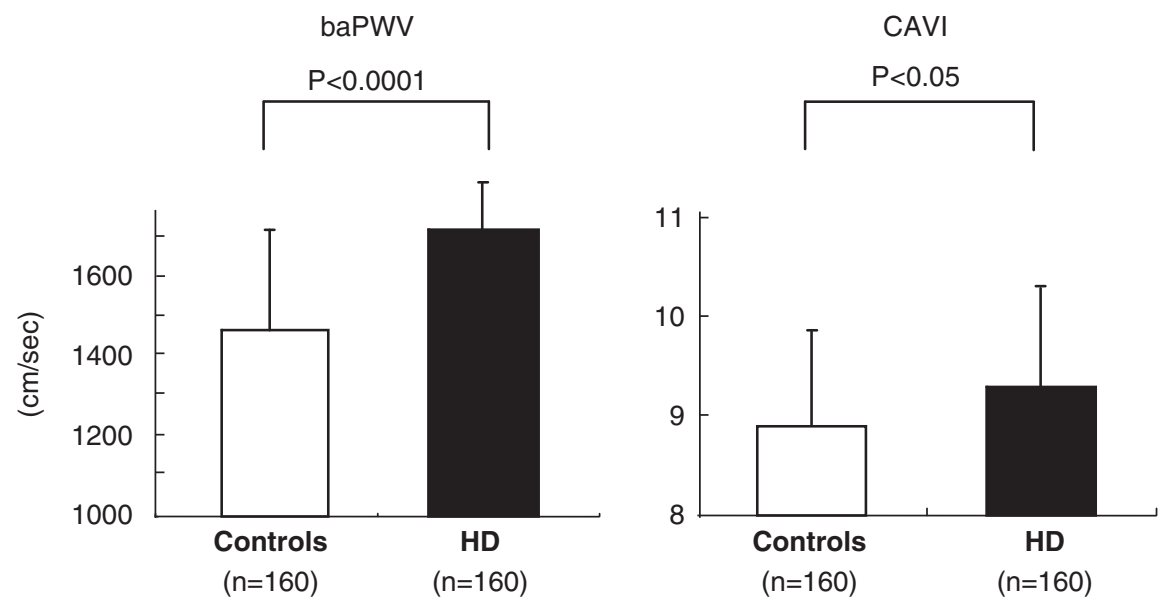

Figure 1 Comparison of baPWV and CAVI between HD patients and controls. baPWV, brachial-ankle pulse wave velocity; CAVI, cardio-ankle vascular index; $\mathrm{HD}$, hemodialysis. 
There was no significant difference in age, gender, HDL-C and TG between the two groups, whereas SBP, DBP and PP were significantly higher and BMI and LDL-C significantly lower in the HD patients compared with the control group. In addition, both baPWV and CAVI were significantly higher in the HD patients than in the control group (Figure 1).

Table 2 Correlation coefficients between baPWV or CAVI and other variables in HD patients calculated by linear regression analysis

\begin{tabular}{lccccc}
\hline & \multicolumn{2}{c}{ baPWV } & & \multicolumn{2}{c}{ CAVI } \\
\cline { 2 - 3 } \cline { 5 - 6 } Variables & $\mathrm{R}$ & $\mathrm{P}$-value & & $\mathrm{R}$ & P-value \\
\hline Age (years) & 0.549 & $<0.0001$ & & 0.562 & $<0.0001$ \\
SBP (mm Hg) & 0.510 & $<0.0001$ & & 0.395 & $<0.0001$ \\
DBP (mm Hg) & 0.203 & $<0.0001$ & & 0.018 & NS \\
PP & 0.499 & $<0.0001$ & & 0.490 & $<0.0001$ \\
Max IMT & 0.285 & $<0.001$ & & 0.280 & $<0.001$ \\
Kt V-1 & 0.221 & $<0.01$ & & 0.216 & $<0.01$ \\
Duration of dialysis & 0.375 & $<0.0001$ & & 0.390 & $<0.0001$ \\
Brinkman index & 0.186 & $<0.05$ & & 0.238 & $<0.01$ \\
BMI & 0.020 & NS & & 0.041 & NS \\
LDL-C & 0.051 & NS & & 0.064 & NS \\
HDL-C & 0.155 & NS & 0.131 & NS \\
TG & 0.067 & NS & 0.001 & NS \\
ip $\times$ Ca & 0.101 & NS & 0.082 & NS \\
i PTH & 0.104 & NS & 0.126 & NS \\
\hline
\end{tabular}

Abbreviations: baPWV, brachial-ankle pulse wave velocity; BMI, body mass index; CAVI, cardioankle vascular index; DBP, diastolic blood pressure; HDL-C, high-density lipoprotein cholesterol; ankle vascular index; DBP, diastolic blood pressure; HDL-C, high-density lipoprotein cho
i PTH, intact parathyroid hormone; LDL-C, low-density lipoprotein cholesterol; Max IMT, i PTH, intact parathyroid hormone; LDL-C, low-density lipoprotein cholesterol; Max IMT,
maximum intima-media thickness; NS, not significant; PP, pulse pressure; SBP, systolic blood pressure; TG, triglyceride.
Linear regression analysis of baPWV or CAVI and other variables in HD patients

The results of the linear regression analysis of baPWV and the other variables in the HD patients are shown in Table 2. Positive correlations were found between baPWV and age, SBP, DBP, PP, $\mathrm{Kt} \mathrm{V}^{-1}$, Brinkman index and max IMT and between CAVI and age, SBP, PP, $\mathrm{Kt} \mathrm{V}^{-1}$, Brinkman index and max IMT. Although baPWV and CAVI showed a similar degree of correlation with age, CAVI was more weakly correlated with SBP than baPWV (Figure 2).

\section{Multiple regression analysis between baPWV or CAVI and other} variables in HD patients

Table 3 shows the results of a multiple regression analysis with baPWV or CAVI as the dependent variable, and age, SBP, PP, max IMT, $\mathrm{Kt} \mathrm{V}^{-1}$, duration of dialysis and diabetes mellitus as the independent variables. In HD patients, baPWV correlated independently with age, SBP and PP, whereas CAVI correlated independently with age, SBP, $\mathrm{Kt}^{-1}$ and diabetes mellitus.

\section{ROC curve analysis between baPWV or CAVI and increased max} IMT $(\geqslant 0.9 \mathrm{~mm})$

Figure 3 demonstrates the ROC curves of baPWV and CAVI to predict increased max IMT $(\geqslant 0.9 \mathrm{~mm})$. The area under the ROC curve (AUC) for baPWV was 0.66, with the highest discriminating sensitivity and specificity being 0.76 and 0.59 , respectively at $\mathrm{baPWV}=1563 \mathrm{~cm} \mathrm{~s}^{-1}$. In contrast, the AUC for CAVI was 0.65 and the highest discriminating sensitivity and specificity were 0.57 and 0.70 , respectively at $\mathrm{CAVI}=9.5$. These results suggest that baPWV and CAVI have similar power to predict the increases in max IMT.

\section{Effect of water volume changes on baPWV and CAVI}

To analyze the effect of HD on baPWV and CAVI, we measured both indices immediately before and after HD in 101 patients (Table 4). In these patients, SBP, DBP and baPWV decreased significantly after HD,
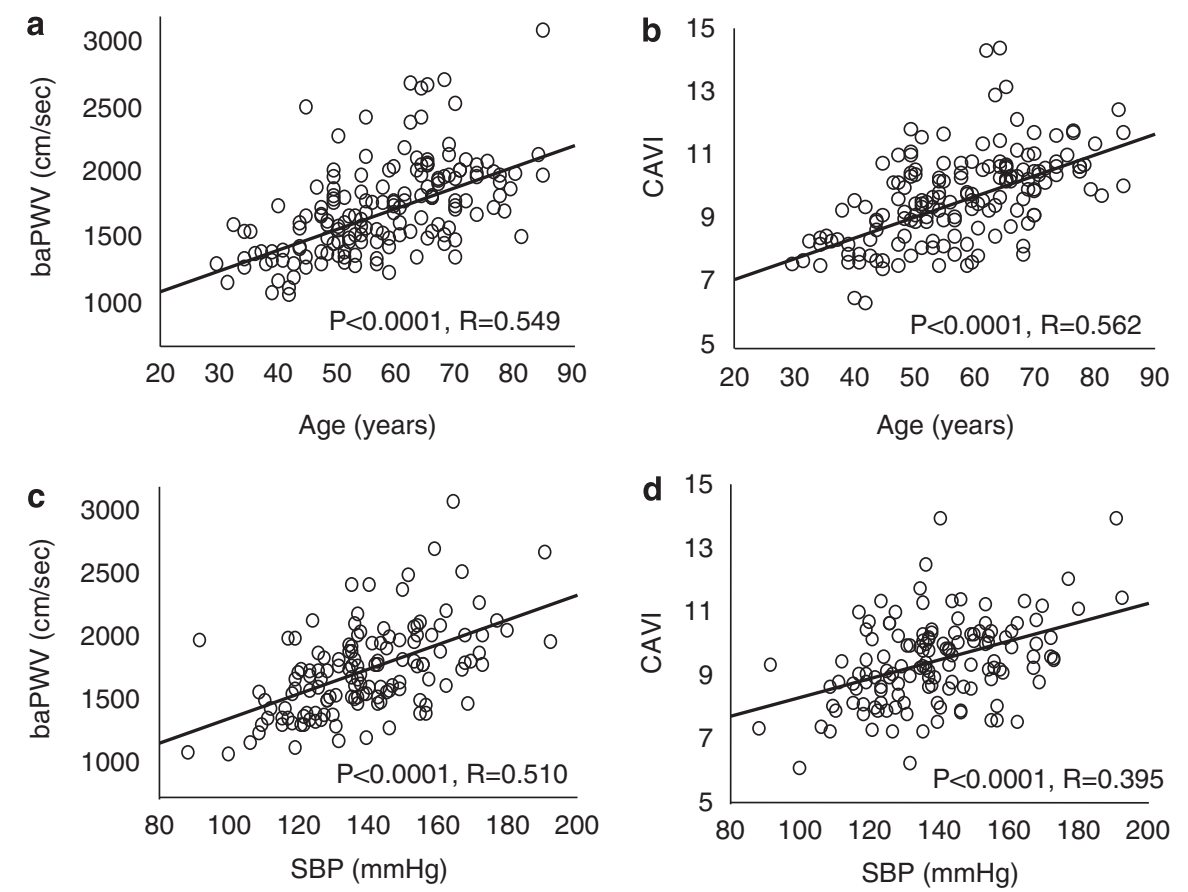

Figure 2 Relationship between baPWV and age (a), CAVI and age (b), baPWV and SBP (c) and CAVI and SBP (d). baPWV, brachial-ankle pulse wave velocity; CAVI, cardio-ankle vascular index; SBP, systolic blood pressure. 
whereas CAVI increased significantly after HD. We also determined the water removal rate as follows; (body weight before HD-body weight after HD)/dry weight. CAVI increased significantly after HD in patients with a water removal rate $>5 \%$, but did not change in patients with a water removal rate $\leqslant 5 \%$. In contrast, baPWV decreased significantly after HD with no influence of water removal rate being observed. These results suggest that CAVI is influenced by changes in water volume.

Table 3 Multiple regression analysis between baPWV or CAVI and other variables in HD patients

\begin{tabular}{|c|c|c|c|c|}
\hline \multirow[b]{2}{*}{ Variable } & \multicolumn{2}{|c|}{ baPWV } & \multicolumn{2}{|c|}{ CAVI } \\
\hline & Coefficient & $\mathrm{P}$-value & Coefficient & P-value \\
\hline Age (years) & 13.987 & $<0.0001$ & 0.056 & $<0.0001$ \\
\hline SBP (mm Hg) & 9.689 & $<0.0001$ & 0.017 & $<0.05$ \\
\hline PP & -8.078 & $<0.05$ & & NS \\
\hline Max IMT & & NS & & NS \\
\hline $\mathrm{KtV}^{-1}$ & & NS & -0.789 & $<0.05$ \\
\hline Duration of dialysis & & NS & & NS \\
\hline HDL-C & & NS & & NS \\
\hline DM & & NS & 0.73 & $<0.05$ \\
\hline
\end{tabular}

Abbreviations: baPWV, brachial-ankle pulse wave velocity; CAVI, cardio-ankle vascular index; $\mathrm{Cr}$, serum creatinine; DM, diabetic mellitus; HDL-C, high density lipoprotein cholesterol; Max IMT, maximum intima-media thickness; PP, pulse pressure; SBP, systolic blood pressure.

\section{DISCUSSION}

This study demonstrated that baPWV and CAVI were both significantly higher in HD patients than in age- and gender-matched controls. In HD patients, baPWV and CAVI showed positive correlations with age, SBP, PP, $\mathrm{KtV}^{-1}$, Brinkman index and max IMT. Multiple regression analysis revealed that baPWV and CAVI were correlated independently with age and SBP. ROC curve analyses demonstrated that baPWV and CAVI had similar power to predict increases in max IMT. Furthermore, we measured baPWV and CAVI immediately before and after HD and showed CAVI was influenced by changes in water volume.

The baPWV is a noninvasive clinical index of arterial stiffness in HD patients. It has been reported that glycated albumin, but not glycated hemoglobin or plasma glucose, is independently and positively associated with baPWV in HD patients with type 2 diabetes. ${ }^{12}$ Kobayashi et al. ${ }^{21}$ also reported a positive association between blood rheology and carotid IMT and baPWV in 118 HD patients. Furthermore, there is evidence to show that baPWV is useful for identifying a high-risk population of $\mathrm{HD}$ patients with an $\mathrm{ABI}$ greater than 0.9. ${ }^{11}$ In addition, it has been reported that baPWV measurement shows variations at different time points in HD patients. ${ }^{22} \mathrm{Su}$ et al. ${ }^{22}$ measured baPWV before and after $\mathrm{HD}$ and then on the next dialysis-free day and found baPWV increased significantly after HD, despite a significant decrease in body weight and BP.

CAVI is a useful index of arterial distensibility ${ }^{16}$ and is not influenced by BP changes during measurement. ${ }^{17}$ Several studies have shown the usefulness of CAVI for detecting atherosclerotic disease, ${ }^{23,24}$ with one study in 67 HD patients using multiple regression analysis to reveal that the high-molecular weight adiponectin to
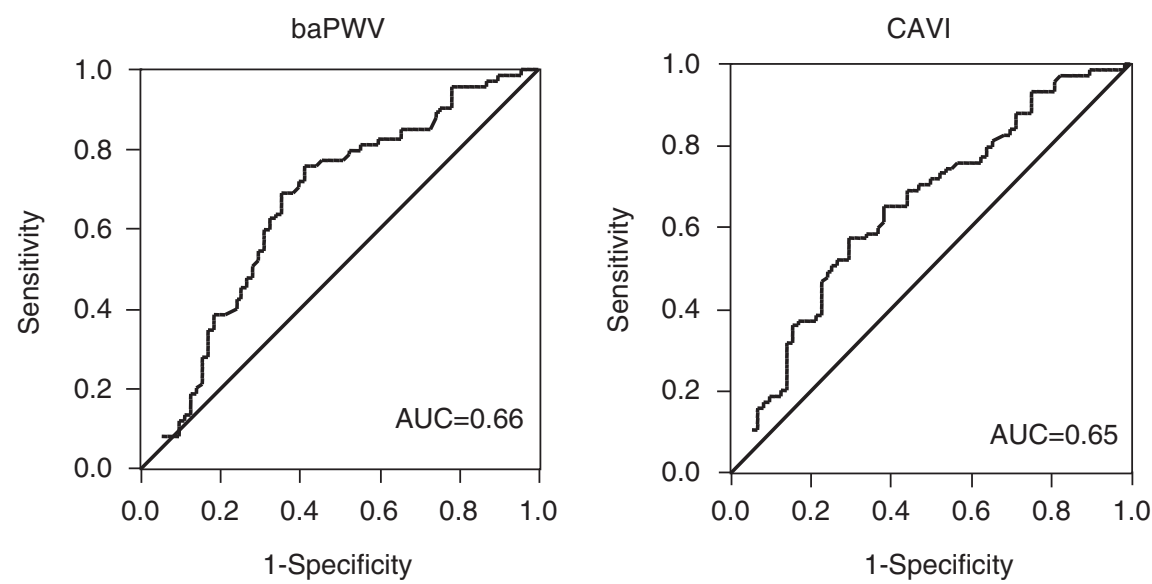

Figure 3 Receiver operating characteristic (ROC) curves of the ability of baPWV and CAVI to predict increases in IMT ( $\geqslant 0.9 \mathrm{~mm})$. AUC, area under ROC curve; baPWV, brachial-ankle pulse wave velocity; CAVI, cardio-ankle vascular index.

Table 4 Effect of changes in water volume on baPWV and CAVI

\begin{tabular}{|c|c|c|c|c|c|c|c|c|c|}
\hline & \multicolumn{3}{|c|}{ Total $(\mathrm{n}=101)$} & \multicolumn{3}{|c|}{ Water removal rate $\leqslant 5 \%(n=33)$} & \multicolumn{3}{|c|}{ Water removal rate $>5 \%(n=68)$} \\
\hline & Before $H D$ & After $H D$ & P-value & Before HD & After $H D$ & P-value & Before $H D$ & After $H D$ & $\mathrm{P}$-value \\
\hline Body weight (kg) & $53.6 \pm 9.9$ & $50.8 \pm 9.6$ & $<0.0001$ & $55.7 \pm 9.2$ & $53.6 \pm 8.9$ & $<0.0001$ & $52.7 \pm 10.1$ & $49.4 \pm 9.6$ & $<0.0001$ \\
\hline $\mathrm{SBP}(\mathrm{mm} \mathrm{Hg})$ & $155 \pm 23$ & $125 \pm 20$ & $<0.0001$ & $144 \pm 21$ & $125 \pm 22$ & $<0.0001$ & $159 \pm 23$ & $125 \pm 20$ & $<0.0001$ \\
\hline $\mathrm{DBP}(\mathrm{mm} \mathrm{Hg})$ & $86 \pm 12$ & $76 \pm 12$ & $<0.0001$ & $81 \pm 10$ & $73 \pm 11$ & $<0.001$ & $89 \pm 11$ & $78 \pm 12$ & $<0.0001$ \\
\hline baPWV $\left(\mathrm{cm} \mathrm{s}^{-1}\right)$ & $1794 \pm 394$ & $1711 \pm 385$ & $<0.05$ & $1740 \pm 334$ & $1656 \pm 320$ & $<0.05$ & $1820 \pm 419$ & $1737 \pm 412$ & $<0.05$ \\
\hline CAVI & $8.9 \pm 1.4$ & $9.4 \pm 1.4$ & $<0.0001$ & $9.3 \pm 1.4$ & $9.4 \pm 1.4$ & NS & $8.7 \pm 1.3$ & $9.4 \pm 1.4$ & $<0.0001$ \\
\hline
\end{tabular}

Abbreviations: baPWV, brachial-ankle pulse wave velocity; CAVI, cardio-ankle vascular index; DBP, diastolic blood pressure; HD, hemodialysis; SBP, systolic blood pressure. 
total adiponectin ratio was an independent determinant of PWV and CAVI. ${ }^{25}$ Takaki et al. ${ }^{26}$ compared baPWV and CAVI in 130 patients with chest pain syndrome and concluded that CAVI was superior to baPWV as a parameter of arterial stiffness. A further study in $103 \mathrm{HD}$ patients used multiple regression analysis to show a significant association between histological arterial fibrosis and CAVI, but no such relationship with PWV. ${ }^{18}$ Ichihara et al. ${ }^{13,14}$ reported that several drugs including an angiotensin II receptor blocker (losartan), angiotensin-converting enzyme-I (tradolapril) and HMG-CoA reductase inhibitor (fluvastatin) improved arterial stiffness measured by PWV. However, in our study, there was no significant difference in baPWV or CAVI in patients either taking or not taking an angiotensin II receptor blocker, angiotensin-converting enzyme-I or HMG-CoA reductase inhibitor (data not shown). The previous studies by Ichihara et al. ${ }^{13,14}$ were longitudinal studies, whereas the present study was a cross-sectional design.

We demonstrated that CAVI increased significantly after HD in patients with a removal rate $>5 \%$. Large volume reduction after HD may increase the sympathetic nervous activity, which increases the vascular tone. The increase of vascular tone may result in the increase of CAVI. In addition, the hemoconcentration after HD may increase the CAVI, because the density of blood $(\rho)$ is one of the determinants of CAVI.

There are several limitations in the measurement of CAVI as it cannot be determined accurately in patients with aortic stenosis, peripheral arterial disease or atrial fibrillation. An ABI $<0.95$ has been reported to be the cutoff value for diminished baPWV accuracy, ${ }^{27}$ and therefore CAVI cannot be measured accurately if the ABI is less than 0.95. Further prospective studies are therefore needed to evaluate the validity of CAVI in the clinical assessment and prediction of mortality in HD patients.

In conclusion, baPWV and CAVI in HD patients were significantly higher than in age- and gender-matched control subjects, with both indices correlating independently with age and SBP in the HD patients. ROC curve analysis demonstrated that the power of baPWV and CAVI to predict increases in max IMT was similar. Furthermore, we measured baPWV and CAVI immediately before and after HD, and showed CAVI was influenced by changes in water volume. Therefore, both baPWV and CAVI are useful indices of arterial stiffness in HD patients.

\section{CONFLICT OF INTEREST}

The authors declare no conflict of interest.

1 Sarnak MJ, Levey AS, Schoolwerth AC, Coresh J, Culleton B, Hamm LL, McCullough PA, Kasiske BL, Kelepouris E, Klag MJ, Parfrey P, Pfeffer M, Raij L, Spinosa DJ, Wilson PW. Kidney disease as a risk factor for development of cardiovascular disease: a statement from the American Heart Association Councils on Kidney in Cardiovascular Disease, High Blood Pressure Research, Clinical Cardiology, and Epidemiology and Prevention. Circulation 2003; 108: 2154-2169.

2 Lysaght MJ. Maintenance dialysis population dynamics: current trends and long-term implications. J Am Soc Nephrol 2002; 13: S37-S40.

3 Shinzato T, Nakai S, Akiba T, Yamagami S, Yamazaki C, Kitaoka T, Kubo K, Maeda K, Morii $\mathrm{H}$. Report of the annual statistical survey of the Japanese Society for Dialysis Therapy in 1996. Kidney Int 1999; 55: 700-712.

4 Ishimitsu T, Nakano N, Sudo Y, Akashiba A, Takahashi T, Ohta S, Minami J, Matsuoka $\mathrm{H}$. Predictive significance of blood pressure values for the incidence of cardiovascular events in chronic hemodialysis patients. Hypertens Res 2008; 31: 1703-1709.
5 O'Hare A, Johansen K. Lower-extremity peripheral arterial disease among patients with end-stage renal disease. J Am Soc Nephrol 2001; 12: 2838-2847.

6 Kubo T, Miyata M, Minagoe S, Setoyama S, Maruyama I, Tei C. A simple oscillometric technique for determining new indices of arterial distensibility. Hypertens Res 2002; 25: 351-358.

7 Yamashina A, Tomiyama H, Takeda K, Tsuda H, Arai T, Hirose K, Koji Y, Hori S, Yamamoto $Y$. Validity, reproducibility, and clinical significance of noninvasive brachialankle pulse wave velocity measurement. Hypertens Res 2002; 25: 359-364.

8 Imanishi R, Seto S, Toda G, Yoshida M, Ohtsuru A, Koide Y, Baba T, Yano K. High brachial-ankle pulse wave velocity is an independent predictor of the presence of coronary artery disease in men. Hypertens Res 2004; 27: 71-78.

9 Nakamura U, Iwase M, Nohara S, Kanai H, Ichikawa K, lida M. Usefulness of brachialankle pulse wave velocity measurement: correlation with abdominal aortic calcification. Hypertens Res 2003; 26: 163-167.

10 Kobayashi K, Akishita M, Yu W, Hashimoto M, Ohni M, Toba K. Interrelationship between non-invasive measurements of atherosclerosis: flow-mediated dilation of brachial artery, carotid intima-media thickness and pulse wave velocity. Atherosclerosis 2004; 173: 13-18.

11 Kitahara T, Ono K, Tsuchida A, Kawai H, Shinohara M, Ishii Y, Koyanagi H, Noguchi T, Matsumoto T, Sekihara T, Watanabe Y, Kanai H, Ishida H, Nojima Y. Impact of brachialankle pulse wave velocity and ankle-brachial blood pressure index on mortality in hemodialysis patients. Am J Kidney Dis 2005; 46: 688-696.

12 Kumeda Y, Inaba M, Shoji S, Ishimura E, Inariba H, Yabe S, Okamura M, Nishizawa Y. Significant correlation of glycated albumin, but not glycated hemoglobin, with arterial stiffening in hemodialysis patients with type 2 diabetes. Clin Endocrinol 2008; 69: 556-561.

13 Ichihara A, Hayashi M, Ryuzaki M, Handa M, Furukawa T, Saruta T. Fluvastatin prevents development of arterial stiffness in hemodialysis patients with type 2 diabetes mellitus. Nephrol Dial Transplant 2002; 17: 1513-1517.

14 Ichihara A, Hayashi M, Kaneshiro Y, Takemitsu T, Homma K, Kanno Y, Yoshizawa M, Furukawa T, Takenaka T, Saruta T. Low doses of Iosartan and trandolapril improve arterial stiffness in hemodialysis patients. Am J Kidney Dis 2005; 45: 866-874.

15 Yambe T, Yoshizawa M, Saijo Y, Yamaguchi T, Shibata M, Konno S, Nitta S, Kuwayama T. Brachio-ankle pulse wave velocity and cardio-ankle vascular index (CAVI). Biomed Pharmacother 2004; 58: S95-S98.

16 Shirai K, Utino J, Otsuka K, Takata M. A novel blood pressure-independent arterial wall stiffness parameter; cardio-ankle vascular index (CAVI). J Atheroscler Thromb 2006; 13: 101-107.

17 Kubozono T, Miyata M, Ueyama K, Nagaki A, Otsuji Y, Kusano K, Kubozono O, Tei C. Clinical significance and reproducibility of new arterial distensibility index. Circ $J$ 2007; 71: 89-94.

18 Ichihara A, Yamashita N, Takemitsu T, Kaneshiro Y, Sakoda M, Kurauchi-Mito A, Itoh H. Cardio-ankle vascular index and ankle pulse wave velocity as a marker of arterial fibrosis in kidney failure treated by hemodialysis. Am J Kidney Dis 2008; 52: 947-955.

19 Sakane K, Miyoshi T, Doi M, Hirohata S, Kaji Y, Kamikawa S, Ogawa H, Hatanaka K, Kitawaki T, Kusachi S, Yamamoto K. Association of new arterial stiffness parameter, the cardio-ankle vascular index, with left ventricular diastolic function. J Atheroscler Thromb 2008; 15: 261-268.

20 Shinzato T, Nakai S, Fujita Y, Takai I, Morita H, Nakane K, Maeda K. Determination of $\mathrm{Kt} / \mathrm{V}$ and protein catabolic rate using pre- and postdialysis blood urea nitrogen concentrations. Nephron 1994; 67: 280-290.

21 Kobayashi S, Okamoto K, Maesato K, Moriya H, Ohtake T. Important role of blood rheology in atherosclerosis of patients with hemodialysis. Hemodial Int 2005; 9: 268-274.

22 Su HM, Chang JM, Lin FH, Chen SC, Voon WC, Cheng KH, Wang CS, Lin TH, Lai WT, Sheu SH. Influence of different measurement time points on brachial-ankle pulse wave velocity and ankle-brachial index in hemodialysis patients. Hypertens Res 2007; 30: 965-970.

23 Nakamura K, Tomaru T, Yamamura S, Miyashita Y, Shirai K, Noike H. Cardio-ankle vascular index is a candidate predictor of coronary atherosclerosis. Circ J 2008; 72: 598-604.

24 Okura T, Watanabe S, Kurata M, Manabe S, Koresawa M, Irita J, Enomoto D, Miyoshi K, Fukuoka T, Higaki J. Relationship between cardio-ankle vascular index (CAVI) and carotid atherosclerosis in patients with essential hypertension. Hypertens Res 2007; 30: 335-340.

25 Kato A, Odamaki M, Ishida J, Hishida A. Association of high-molecular-weight to total adiponectin ratio with pulse wave velocity in hemodialysis patients. Nephron Clin Prac 2008; 109: c18-c24

26 Takaki A, Ogawa H, Wakeyama T, Iwami T, Kimura M, Hadano Y, Matsuda S, Miyazaki Y, Hiratsuka A, Matsuzaki M. Cardio-ankle vascular index is superior to brachial ankle pulse wave velocity as an index of arterial stiffness. Hypertens Res 2008; 31: 13471355

27 Motobe K, Tomiyama H, Koji Y, Yambe M, Gulinisa Z, Arai T, Ichihashi H, Nagae T, Ishimaru S, Yamashina A. Cut-off value of the ankle-brachial pressure index at which the accuracy of brachial-ankle pulse wave velocity measurements is diminished. Circ J 2005; 69: 55-60. 